\title{
ANTIARRHYTHMIC AND ELECTROPHYSIOLOGIC ACTIONS OF CLOFILIUM IN EXPERIMENTAL CANINE MODELS ${ }^{1}$
}

\author{
GREGORY A. KOPIA ${ }^{2,3}$, BRIAN T. ELLER, EUGENE PATTERSON, MICHAEL J. SHEA ${ }^{2}$ and BENEDICT R. LUCCHESI ${ }^{4}$ \\ Department of Pharmacology, The University of Michigan Medical School, Ann Arbor, Michigan 48109, U.S.A.
}

Received 21 March 1985, revised MS received 25 June 1985, accepted 9 July 1985

G.A. KOPIA, B.T. ELLER, E. PATTERSON, M.J. SHEA and B.R. LUCCHESI, Antiarrhythmic and electrophysiologic actions of clofilium in experimental canine models, European J. Pharmacol. 116 (1985) 49-61.

Clofilium was studied in three experimental models. In non-ischemic and chronically infarcted canine hearts, clofilium $(0.5-2 \mathrm{mg} / \mathrm{kg}$ ) produced a dose-dependent increase in electrical ventricular fibrillation threshold (VFT), but prolonged the effective refractory period (ERP) of normal myocardium in only the non-ischemic heart. When chronically infarcted hearts were subjected to programmed electrical stimulation, $1 \mathrm{mg} / \mathrm{kg}$ of clofilium inhibited the re-induction of either ventricular tachycardia or ventricular fibrillation in 5 of 6 animals and slowed the rate of the induced tachycardia in the sixth. Clofilium, however, failed to alter ventricular refractory periods of normal myocardium at either twice diastolic threshold current ( $176 \pm 5 \mathrm{~ms}$ control vs. $187 \pm 9 \mathrm{~ms}$ post-clofilium, $\mathrm{P}>0.05)$ or at $10 \mathrm{~mA}(134 \pm 6 \mathrm{~ms}$ control vs. $137 \pm 13 \mathrm{~ms}$ post-clofilium, $\mathbf{P}>0.05)$. In addition, chronic administration of clofilium ( $2 \mathrm{mg} / \mathrm{kg}$, i.v., followed by $1 \mathrm{mg} / \mathrm{kg}$ every $12 \mathrm{~h})$ was ineffective in decreasing mortality in a canine model of sudden coronary death. Of 10 saline-treated conscious animals subjected to an electrically-induced intimal lesion of the left circumflex coronary artery in the presence of a previous ischemic insult, all 10 died suddenly of ventricular fibrillation within $173 \pm 45 \mathrm{~min}$ after current application. Under similar conditions, 7 clofilium-treated animals died suddenly within $249 \pm 88 \mathrm{~min}(\mathrm{P}>0.05)$ after current application while 3 animals survived $(\mathrm{P}>0.10)$. Clofilium did, however, elevate the effective refractory period in these animals $(150 \pm 3 \mathrm{~ms}$ saline-treated vs. $195 \pm 7 \mathrm{~ms}$ clofiliumtreated). It is concluded from our data that there is little relationship between clofilium's electrophysiologic actions in normal myocardium and antiarrhythmic effects. Furthermore, simple prolongation of refractoriness in normal non-ischemic myocardium may be insufficient for the prevention of ventricular fibrillation which develops in response to a transient ischemic event superimposed on a chronically injured myocardium.

Clofilium Ventricular fibrillation Ventricular tachycardia Sudden coronary death

\footnotetext{
${ }^{1}$ Supported by NIH Grant No. HL-05806-23. Portions of this work have been presented previously at the 65 th annual meeting of the Federation of American Societies for Experimental Biology (Fed. Proc. 40: 673, 1981) and the 54th Scientific Sessions of the American Heart Association (Circulation 64: 124, 1981).

2 This work was completed while G.A.K. and M.J.S. were Fellows of the Michigan Heart Association.

${ }^{3}$ Current address: Smith, Kline and French Laboratories, 1500 Spring Garden Street, Philadelphia, PA 19101, U.S.A.

4 To whom all correspondence should be addressed: Department of Pharmacology, M6322 Medical Science Building I, The University of Michigan Medical School, Ann Arbor, Michigan 48109, U.S.A.
}

\section{Introduction}

There has been recent interest in the development and use of pharmacologic agents for the prevention of sudden coronary death. The $\beta$ adrenergic receptor antagonists timolol (Norwegian Multicenter Study Group, 1981), metoprolol (Hjalmarson et al., 1981), and propranolol (BetaBlocker Heart Attack Study Group, 1981) have been reported to reduce the risk of sudden coronary death after myocardial infarction. The quaternary ammonium adrenergic blocking agent bretylium is 
effective in preventing ventricular fibrillation in both experimental animals (Bacaner, 1968; Bacaner and Schrienmachers, 1968; Kniffen et al., 1975; Cervoni et al., 1971) and man (Holder et al., 1977; Sanna and Arcidiacono, 1973). Another quaternary ammonium agent, pranolium, has been reported to reduce the incidence of ventricular fibrillation in experimental animals (Eller et al., 1983). Clofilium, a third drug of the quaternary ammonium type with electrophysiologic and potential antifibrillatory actions, has been investigated recently (Steinberg and Molloy, 1979; Steinberg et al., 1981). Additional electrophysiologic studies using this drug are appropriate to correlate clofilium's reported in vitro actions and its potential in vivo antiarrhythmic and antifibrillatory effects.

In this report we examine the effect of clofilium on: (1) the ventricular fibrillation threshold in an anesthetized, previously infarcted canine preparation; (2) programmed electrical stimulation in anesthetized, previously infarcted dogs; and (3) ventricular fibrillation in a conscious canine model of sudden coronary death.

\section{Materials and methods}

\subsection{Production of myocardial infarction}

Male mongrel dogs were anesthetized with intravenous (i.v.) pentobarbital $(30 \mathrm{mg} / \mathrm{kg})$, intubated, and ventilated with room air. The heart was suspended in a pericardial cradle after exposure by means of a left lateral thoracotomy at the fifth intercostal space. The left anterior descending coronary artery (LAD) was separated from the surrounding tissue at or just below the level of the tip of the left atrial appendage. A critical stenosis was produced by tying a ligature around both the vessel and a $19 \mathrm{~g}$ needle and then withdrawing the latter. The vessel was then occluded using a snare formed from a loop of silastic tubing passed through a flared polyethylene tube. After 90 min of occlusion, the snare was released allowing reperfusion of the vessel through the critical stenosis. The pericardium was then loosely tied, the chest incision closed, and the dogs allowed to recover. Appropriate antibiotic therapy was administered during the recovery phase.

\subsection{Determination of the ventricular fibrillation threshold (VFT)}

Seven normal and seven previously infarcted (4-7 days) male mongrel dogs weighing between 12 and $14 \mathrm{~kg}$ were anesthetized with sodium pentobarbital ( $30 \mathrm{mg} / \mathrm{kg}$ i.v.), intubated, and ventilated with room air at a rate and tidal volume which maintained normal blood gases and $\mathrm{pH}$. The heart was exposed via a left thoracotomy at the fourth or fifth intercostal space and suspended in a pericardial cradle. An acrylic plaque containing 2 silver-silver chloride electrodes $(1 \mathrm{~mm}$ diameter, 3 $\mathrm{mm}$ apart) were sewn to the surface of the heart in the interventricular septum adjacent to the right ventricular outflow tract for the determination of VFT. A second acrylic plaque electrode was sewn to the heart in an area of tissue perfused by the left circumflex coronary artery. This electrode was used for the determination of the excitation threshold and the Q-EG interval (time in ms from the beginning of the Q-wave to the peak of the ventricular electrogram, i.e., conduction time or CT). A bipolar plunge electrode (insulated stainless steel $25 \mathrm{~g}$ wire, $4 \mathrm{~mm}$ long, $2 \mathrm{~mm}$ apart) was placed high in the left ventricle for determination of the effective refractory period (ERP). For all VFT studies, the ERP was measured at twice diastolic threshold current. In addition, a small bipolar plaque electrode was attached to the left atrial appendage for left atrial pacing. All electrophysiologic measurements were made in normal, non-infarcted tissue regardless of whether or not the animal had previously undergone myocardial infarction. For most experiments, the heart was paced at a constant rate during the measurement of the electrophysiologic parameters

VFT was measured by delivering electrical current to the heart through the VFT electrode using a Grass S88 stimulator coupled to a Grass CCU-1A constant current unit and an SIU-5 stimulus isolation unit. A Tektronix model 565 oscilloscope with a type $3 \mathrm{~A} 8$ operational amplifier was used to trigger the ventricular stimulus from the peak of the R-wave of the Lead II ECG. The triggering stimulus was delayed $50 \mathrm{~ms}$ after $\mathrm{R}$ activation so that the impulse train scanned the T-wave. Current was delivered every 6-8 beats using a $200 \mathrm{~ms}$ 
train of $4 \mathrm{~ms}$ pulses at a frequency of $60 \mathrm{~Hz}$. Each current level was tested 3 times before moving on to the next highest current. An increasing amount of current was given until the animal fibrillated, at which time defibrillation was accomplished within 15-20 s using a Physio-control series 70 DC defibrillator.

Blood pressure via a cannulated carotid artery and the Lead II ECG were monitored continuously on a Grass model 7 polygraph. Drug injections were made through a cannulated external jugular vein.

For the determination of the Q-EG interval, both the Lead II ECG and the ventricular electrogram were displayed at high sweep speed (10 $\mathrm{ms}$ /division) on a Tektronix 5111 storage oscilloscope and the time between waveforms measured.

After two control determinations (separated by $1 \mathrm{~h}$ ) of all parameters each dog received sequential and cumulative doses of $0.5,1.0$ and $2.0 \mathrm{mg} / \mathrm{kg}$ of clofilium i.v., each dose being separated by $1 \mathrm{~h}$. One $h$ after drug administration the electrophysiological parameters were measured again prior to administration of the next higher dose. After the last VFT determination, animals were not defibrillated and the experiment was concluded.

\subsection{Programmed electrical stimulation}

Seven dogs weighing between 12 and $17 \mathrm{~kg}$ were infarcted as described above and allowed to recover for 4 to 7 days. On the day of the experiment, each dog was reanesthetized with $30 \mathrm{mg} / \mathrm{kg}$ of sodium pentobarbital, intubated, and mechanically ventilated with room air. Cannulae were placed in the left common carotid artery for measurement of blood pressure and in the left external jugular vein for injection of drugs. Blood pressure, Lead II ECG, and heart rate derived from the ECG were monitored continuously throughout the experiment on a Grass model 7 polygraph and the ECG was stored on magnetic tape (Lockheed Store 4D) for later analysis. The chest was opened at the fourth or fifth intercostal space, the heart exposed and suspended in a pericardial cradle. Programmed electrical stimulation was performed in a manner similar to that of Michelson et al. (1980). Briefly, a single plunge wire electrode $(0.1 \mathrm{~mm}$ diameter silver wire, Teflon coated except at the tip) was inserted into the left ventricle in the area of the right ventricular outflow tract at, or slightly below, the level of the previous LAD occlusion in normal tissue at the border of the region of infarction. The electrode was connected to the cathode of a Grass constant current unit (CCU-1A) which was in turn connected to the $S_{2}$ output of a Grass S88 stimulator through a stimulus isolation unit (Grass SIU-5). The anode of the constant current unit was connected to the rib retractors which provided a contact surface area of $23 \mathrm{~cm}^{2}$. A Tektronix model 565 oscilloscope with type $3 \mathrm{~A} 8$ operational amplifier was used to trigger the ventricular stimulus from the R-wave of the Lead II ECG. Using this system, 1, 2 or 3 premature stimuli $(4 \mathrm{~ms})$ could be inserted at timed intervals after the $R$ wave.

Each dog was scanned initially with 3 premature beats at twice diastolic threshold current with $\mathrm{R}-\mathrm{S}_{2}$ intervals ranging from $350 \mathrm{~ms}$ to the refractory period and $S_{2}-S_{3}: S_{3}-S_{4}$ intervals ranging from 200 to $125 \mathrm{~ms}$. If sustained ventricular tachycardia (VT) or ventricular fibrillation could not be induced, the cathodal electrode was moved down the LAD distribution toward the apex of the heart or laterally across the surface of the left ventricle at the level of the LAD occlusion. Most dogs (4) required testing of only 1 site but no more than 6 sites were required in any $\operatorname{dog}(1 \mathrm{dog})$. Once an arrhythmia could be induced in a dog, the strength interval relationship for that site was determined. The current was set at diastolic threshold and the interval was reduced from $300 \mathrm{~ms}$ in $10 \mathrm{~ms}$ increments until the stimulus failed to result in a propagated beat. Then the current was increased until a propagated response could once again be obtained, after which the interval was decreased in 5 $\mathrm{ms}$ increments. Current was increased in $0.01 \mathrm{~mA}$ increments from threshold to $0.1 \mathrm{~mA}$, in $0.05 \mathrm{~mA}$ increments from 0.1 to $1 \mathrm{~mA}$, and in $0.5 \mathrm{~mA}$ increments from 1 to $10 \mathrm{~mA}$. Data are presented as the refractory periods at both twice diastolic threshold current and at $10 \mathrm{~mA}$. In both cases the value represents the longest interval at that particular current that failed to result in a propagated response.

After determining the strength interval relation- 
ship, the stimulus current was reset to twice diastolic threshold and the precise parameters for incuding each animal determined. Once the arrhythmia could be induced reproducibly (at least 3 times), the animal was given $1 \mathrm{ml}$ of $0.9 \% \mathrm{NaCl}$ (saline) for each $\mathrm{kg}$ of body weight and allowed to stabilize for $45 \mathrm{~min}$ to $1 \mathrm{~h}$. The ability to induce the arrhythmia was then re-tested, after which each animal was given clofilium, $1 \mathrm{mg} / \mathrm{kg}$ i.v. (in saline, $\mathrm{pH}$ 7.3-7.4), and again allowed to stabilize for $45 \mathrm{~min}$ to $1 \mathrm{~h}$. The strength interval relationship was then redetermined and the animals retested for inducibility of the arrhythmia. Some animals were tested further at current intensities greater than twice diastolic threshold and with subsequent doses of clofilium.

At the termination of the experiment, each animal was sacrificed by excision of the heart. Cannulae were then inserted in the left anterior descending coronary artery at the site of the previous occlusion and into the root of the aorta. The hearts were then stained by perfusing, at equal pressure $(100 \mathrm{mmHg})$, Evans blue $(0.5 \% \mathrm{w} / \mathrm{v})$ through the aortic root and triphenyltetrazolium $(1 \% \mathrm{w} / \mathrm{v})$ in $0.01 \mathrm{M}$ potassium phosphate buffer into the LAD. After rinsing with tap water, the hearts were sliced in bread-loaf fashion from apex to base and the amount of infarcted tissue (unstained), the tissue at risk of infarction (stained brick red), and the remaining left ventricular mass (stained blue), were determined gravimetrically.

\subsection{Canine model of sudden coronary death}

The antifibrillatory action of clofilium was studied in a new, recently described, canine model of sudden coronary death (Patterson et al., 1982). Briefly, myocardial infarction was produced as described above in 20 dogs weighing between 18 and $22 \mathrm{~kg}$. During the surgical procedure, the left circumflex coronary artery was exposed approximately $1 \mathrm{~cm}$ from its origin and the $3 \mathrm{~mm}$ tip of a $25 \mathrm{~g}$ needle which had previously been attached to a length of $30 \mathrm{~g}$ silver wire, was inserted into the lumen of the artery. The wire was then secured to the epicardial surface of the heart with 3-0 suture. A bipolar plunge electrode $(25 \mathrm{~g}$ insulated stainless steel, $4 \mathrm{~mm}$ long and $3 \mathrm{~mm}$ apart) was inserted into the interventricular septum in the area of the right ventricular outflow tract, approximately $1 \mathrm{~cm}$ above the level of the LAD occlusion in an area bordering the region of infarct. Silver disc electrodes were implanted subcutaneously (s.c.) to approximate locations for recording the Lead II ECG. A cannula was inserted into the left jugular vein. The chest incision was closed and the animals allowed to recover. The ECG of each dog was monitored daily and when sinus rhythm resumed (usually 48-72 $\mathrm{h}$ after surgery) animals were randomized to either control or clofilium treatment groups. Control animals received $10 \mathrm{ml}$ saline and clofilium-treated animals received an initial i.v. dose of $2 \mathrm{mg} / \mathrm{kg}$ followed by additional doses of 1 $\mathrm{mg} / \mathrm{kg}$ every $12 \mathrm{~h}$. Programmed electrical stimulation was performed after $24 \mathrm{~h}$ of treatment, just prior to the next scheduled dose. Animals were studied while conscious and resting comfortably in a sling. One, two or three premature ventricular stimuli $(4 \mathrm{~ms}$ pulses at twice diastolic threshold voltage) were introduced via the electrode in the interventricular septum in a manner similar to that described above. The procedure was performed until non-sustained ventricular tachycardia (selfterminating ventricular tachycardia lasting longer than 3 beats) or sustained ventricular tachycardia was produced in triplicate. A rigorous atempt at production of inducible arrhythmias was avoided in order to circumvent the induction of ventricular fibrillation.

After completion of programmed electrical stimulation, the scheduled dose of clofilium (1 $\mathrm{mg} / \mathrm{kg}$ ) or saline $(10 \mathrm{ml})$ was administered. An anodal current of $150 \mu \mathrm{A}$ was then applied to the intimal surface of the left circumflex coronary artery by connecting the previously implanted wire to a $9 \mathrm{~V}$ battery and a variable potentiometer. The Lead II ECG was recorded via radiotelemetry on a Grass model 7 polygraph. Throughout the duration of intimal stimulation, the animals were ambulatory as all the necessary equipment was secured in the pouch of a nylon mesh vest.

At the time of ventricular fibrillation or the 24 $h$ end point, animals were anesthetized and their hearts were excised. The left circumflex coronary artery was examined for the presence of intimal damage and thrombus formation. Hearts were then 
sectioned in bread-loaf fashion from apex to base and incubated at $37^{\circ} \mathrm{C}$ in $0.5 \%$ triphenyltetrazolium in $0.01 \mathrm{M}$ phosphate buffer, $\mathrm{pH}$ 7.4. Infarct size was determined gravimetrically and expressed as a percentage of the total left ventricular mass.

The Unit for Laboratory and Animal Medicine of the University of Michigan observed and approved the experimental protocols. Experiments were performed according to the 'Guiding Principles in the Care of Laboratory Animals' as outlined by the American Physiological Society.

\subsection{Data analysis}

Data are expressed as the means \pm S.E.M. Differences between doses of clofilium in the VFT study were assessed using 2-way ANOVA and Scheffe's test (Dixon and Massey, 1969). In the programmed electrical stimulation study, differences in electrophysiological parameters after clofilium administration were analyzed using the paired t-test. Fisher's exact test was used to determine whether there was a difference in survival between clofilium and saline-treated animals in the sudden death experiments and Student's unpaired $t$-test was used to determine significant differences between means of saline-treated and clofiliumtreated groups of animals. For all tests, the null hypothesis was rejected only if the probability of the calculated statistic was less than 0.05 .

\section{Results}

\subsection{Effect of clofilium on electrical ventricular fibril- lation threshold}

Figures 1 and 2 summarize the effect of $0.5,1$ and $2 \mathrm{mg} / \mathrm{kg}$ doses of clofilium on both VFT and the effective refractory period in normal and chronically infarcted canine hearts. In normal, non-infarcted hearts, clofilium produced a maximum and sustained elevation in effective refractory period with the smallest dose $(0.5 \mathrm{mg} / \mathrm{kg})$, but failed to increase VFT significantly until after administration of the highest dose $(2.0 \mathrm{mg} / \mathrm{kg}$, fig. 1). Conversely, in the chronically infarcted heart, clofilium failed to significantly increase the effec-

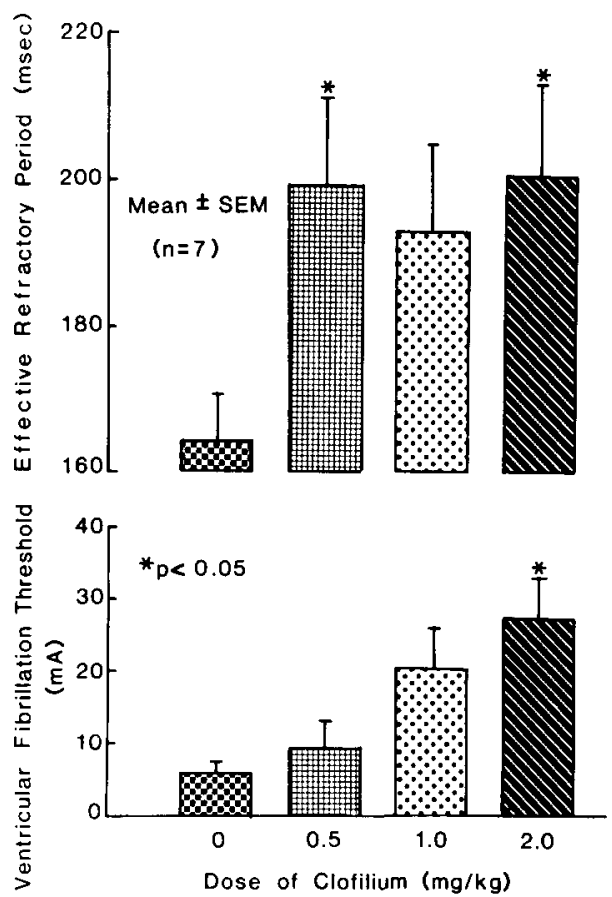

Fig. 1. Antifibrillatory action of clofilium $(0,0.5,1.0$, and 2.0 $\mathrm{mg} / \mathrm{kg}$ ) in the normal non-ischemic canine heart. The upper panel depicts the changes in the effective refractory period (in ms) at twice threshold current after a control determination and after the three doses of clofilium. The bottom panel shows the elevation in electrical VFT produced by clofilium. ${ }^{*}$ Signifies significant difference from control.

tive refractory period at any dose level while producing a graded increase in VFT which achieved statistical significance only at the $2 \mathrm{mg} / \mathrm{kg}$ dose (fig. 2). Control VFT's for both groups were not different $(6.4 \pm 1.7 \mathrm{~mA}$ non-infarcted VFT vs. 9.8 $\pm 3.2 \mathrm{~mA}$ infarcted VFT) nor were the maximum VFT's after the $2 \mathrm{mg} / \mathrm{kg}$ dose of clofilium (28.4 \pm $6.4 \mathrm{~mA}$ non-infarcted VFT vs. $26.2 \pm \mathrm{mA}$ infarcted VFT). Figure 3 is an example of a ventricular fibrillation threshold experiment conducted in a dog subject to previous myocardial infarction.

Table 1 summarizes the remaining electrophysiological and cardiovascular actions of clofilium that were measured in this study. With the exception of a modest decrease in heart rate in the non-infarcted animals, clofilium failed to produce any alterations in mean blood pressure, excitation threshold or conduction time. 


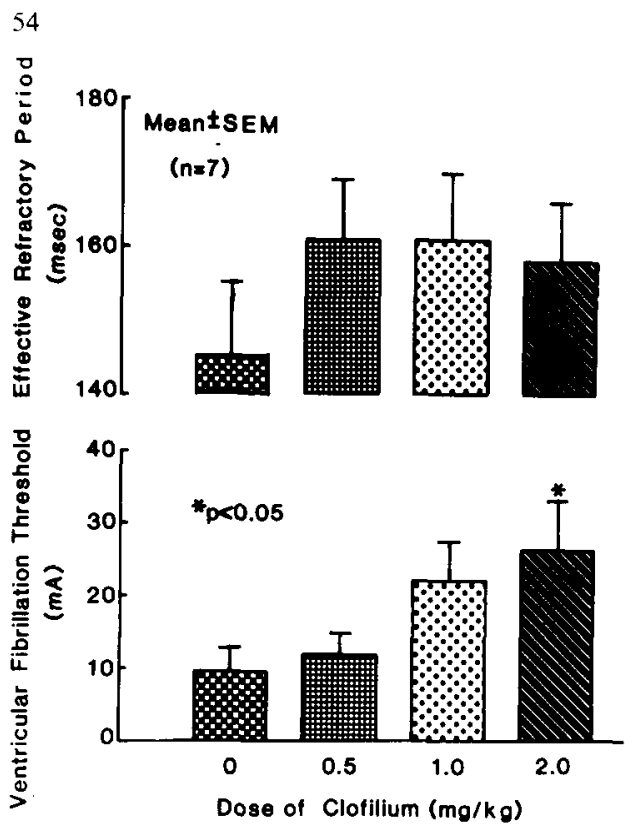

Fig. 2. Antifibrillatory action of clofilium in the chronically infarcted canine heart. Presentation of data similar to that in fig. 1.

\subsection{Effect of clofilium in dogs subject to pro- grammed electrical stimulation}

Tables 2 and 3 summarize the effect of clofilium in a canine model of programmed electrical stimulation. Of the $7 \mathrm{dogs}$ originally studied, 1 animal fibrillated during determination of the strength interval relationship after clofilium administration and could not be revived despite vigorous direct heart massage and repeated cardioversion attempts. This animal is not included in the data of tables 2 and 3. Data from the remaining 6 animals, however, show that administration of $1 \mathrm{mg} / \mathrm{kg}$ of clofilium was effective in either preventing the induction of ventricular tachycardia by programmed electrical stimulation (5 dogs) or slowing the cycle length of the tachycardia ( 1 dog, table 2$)$. This dose of clofilium, however, failed to alter excitation threshold or the effective refractory period at either twice diastolic threshold current or at $10 \mathrm{~mA}$ (table 3). Only 1 animal showed a substantial increase in the effective refractory period (table $3, \operatorname{dog} 1$ ) while 1 animal showed a small decrease in the effective refractory period at twice threshold current and a larger decrease at 10 $\mathrm{mA}$ (table 3, animal 6). The remaining animals showed only minor alterations in refractoriness.

After blocking the induced arrhythmia with 1 $\mathrm{mg} / \mathrm{kg}$ of clofilium, 2 dogs (nos. 2 and 4) were tested further at 5 times diastolic threshold current and were found to be non-inducible even at the increased stimulation current. Two other animals (nos. 1 and 5) were tested at 5 times diastolic threshold current. In animal number 5,3 premature beats at 5 times diastolic threshold current and with coupling intervals of 170,125 and $125 \mathrm{~ms}$ produced a ventricular tachycardia with a cycle length of $138 \mathrm{~ms}$ which rapidly degenerated into

TABLE 1

Electrophysiologic and cardiovascular actions of clofilium in normal and chronically infarcted dogs.

\begin{tabular}{|c|c|c|c|c|c|}
\hline & & \multicolumn{4}{|c|}{ Dose of clofilium $(\mathrm{mg} / \mathrm{kg})$} \\
\hline & & $\overline{0}$ & 0.5 & 1 & 2 \\
\hline Mean blood & $\mathbf{N}$ & $92 \pm 5^{a}$ & $82 \pm 6$ & $82 \pm 7$ & $76 \pm 12$ \\
\hline $\begin{array}{l}\text { pressure } \\
\text { (mmHg) }\end{array}$ & $\mathbf{I}$ & $87 \pm 6$ & $86 \pm 4$ & $80 \pm 3$ & $83 \pm 3$ \\
\hline Heart rate & $\mathbf{N}$ & $158 \pm 9$ & $133 \pm 14^{b}$ & $121 \pm 14^{b}$ & $112 \pm 17^{\mathrm{h}}$ \\
\hline (BPM) & $\mathbf{I}$ & $172 \pm 8$ & $165 \pm 5$ & $154 \pm 7^{2}$ & $165 \pm 5$ \\
\hline Excitation & $\mathrm{N}$ & $0.097 \pm 0.019$ & $0.254 \pm 0.129$ & $0.151 \pm 0.039$ & $0.302 \pm 0.176$ \\
\hline $\begin{array}{l}\text { threshold } \\
\text { (mA) }\end{array}$ & I & $0.6 \pm 0.1$ & $2.1 \pm 1.1$ & $2.0 \pm 1.0$ & $1.5 \pm 0.4$ \\
\hline Conduction & $\mathbf{N}$ & $20 \pm 2$ & $19 \pm 2$ & $21 \pm 2$ & $20 \pm 2$ \\
\hline $\begin{array}{l}\text { time } \\
\text { (ms) }\end{array}$ & $\mathbf{I}$ & $31 \pm 1$ & $31 \pm 1$ & $30 \pm 1$ & $31 \pm 1$ \\
\hline
\end{tabular}

\footnotetext{
${ }^{a}$ Mean \pm S.E.M., $n=7$ for both normal (N) and chronically infarcted (I) groups. ${ }^{b}$ Significantly less than without clofilium; $P<0.05$ by 2-way ANOVA and Scheffe's test.
} 
VENTRICULAR FIBAILLATION THRESHOLD AFTER CLOFLIUM

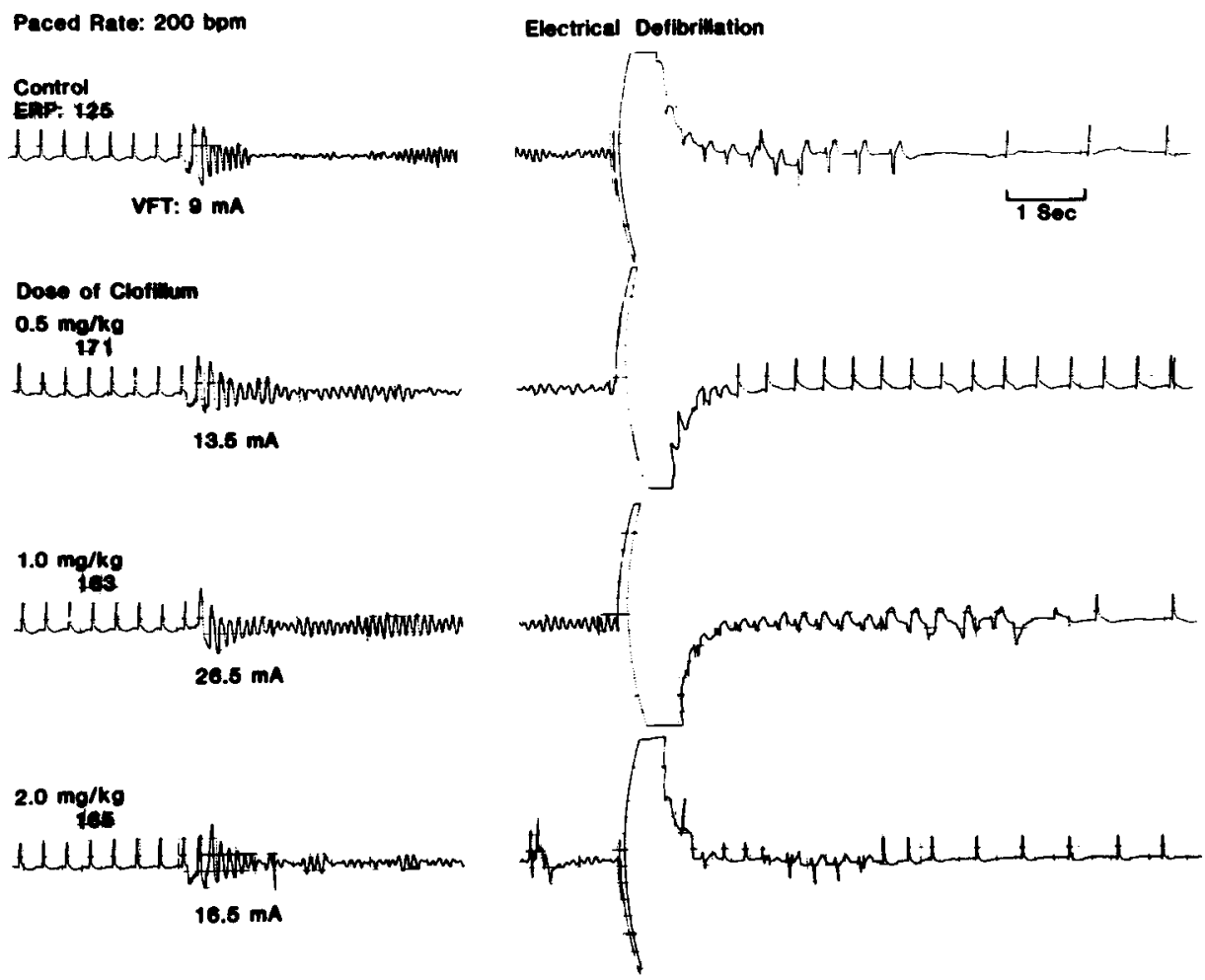

Fig. 3. An example of the antifibrillatory action of clofilium in a chronically infarcted canine heart. In this animal, $1.0 \mathrm{mg} / \mathrm{kg}$ of clofilium produced the largest increase in VFT to a value of $26.5 \mathrm{~mA}$ while the $0.5 \mathrm{mg} / \mathrm{kg}$ dose produced the largest increase in the ERP.

ventricular fibrillation. Further administration of 2 $\mathrm{mg} / \mathrm{kg}$ of clofilium to this dog failed to prevent induction of the ventricular tachycardia, but increased the cycle length to $153 \mathrm{~ms}$ and prevented the degeneration of the ventricular tachycardia to ventricular fibrillation. The remaining animal (no. 1) responded to programmed electrical stimulation at 5 times threshold current and with 3 ventricular stimuli $(230,155,155 \mathrm{~ms})$ leading to ventricular tachycardia which degenerated into ventricular fibrillation. This dog received no further clofilium. However, prior to clofilium, and at twice threshold current, this animal required only 2 premature stimuli at coupling intervals of 180 and $165 \mathrm{~ms}$. After $1 \mathrm{mg} / \mathrm{kg}$ of clofilium even 3 premature stimuli at twice threshold current and at intervals of 230,140 and $140 \mathrm{~ms}$ failed to induce a ventricular tachycardia so that clofilium afforded this animal a considerable degree of protection against the production of the tachycardia

Staining of the hearts from all 7 dogs revealed mottled, non-confluent subendocardial infarcts that occasionally extended transmurally. Average

TABLE 2

Antiarrhythmic actions of clofilium in dogs subject to programmed electrical stimulation.

\begin{tabular}{llllll}
\hline Dog & Pre-clofilium & & \multicolumn{2}{l}{ Clofilium $(1 \mathrm{mg} / \mathrm{kg})$} \\
\cline { 3 - 3 } \cline { 5 - 5 } & Arrhythmia & $\begin{array}{l}\text { Cycle } \\
\text { length (ms) }\end{array}$ & & Arrhythmia & $\begin{array}{l}\text { Cycle } \\
\text { length (ms) }\end{array}$ \\
\hline 1 & V-Tach $^{\text {a }}$ & 200 & & Non-inducible & - \\
2 & V-Tach Fib $^{\text {a }}$ & 125 & & Non-inducible & - \\
3 & V-Tach $_{4}$ & 168 & & Non-inducible & - \\
4 & V-Tach & 158 & & Non-inducible & - \\
5 & V-Tach Fib & 109 & & Non-inducible & - \\
6 & V-Tach & 183 & V-Tach & 203
\end{tabular}

a V-Tach, sustained ventricular tachycardia; Fib, fibrillation. 


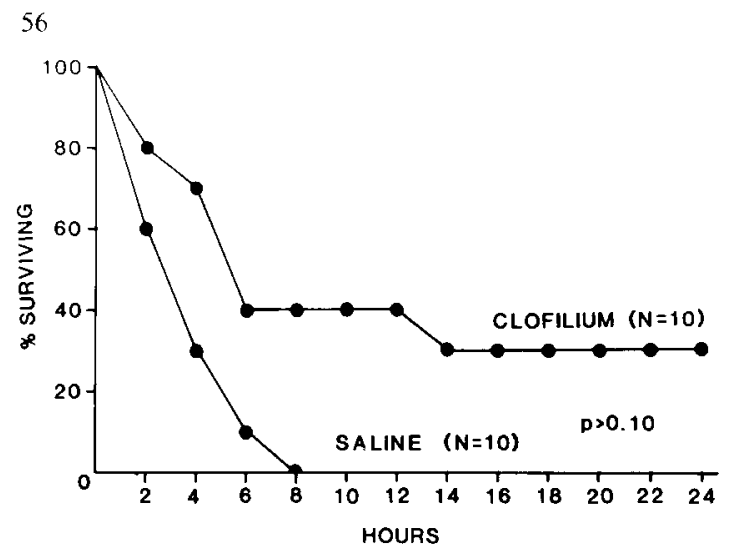

Fig. 4. Effect of clofilium on survival in a canine model of sudden coronary death. Clofilium failed to exert any significant protection against sudden death in this animal model when administered as a $2 \mathrm{mg} / \mathrm{kg}$ loading dose followed by $1 \mathrm{mg} / \mathrm{kg}$ every $12 \mathrm{~h}$ for $24 \mathrm{~h}$. Data were analyzed using Fisher's exact test.

infarct size in these animals was $17.4 \pm 1.9 \%$ of the total left ventricle. The single animal that fibrillated and could not be revived had the largest infarct of any animal: $25.8 \%$ of the left ventricle.

\subsection{Effect of clofilium in a canine model of sudden coronary death}

In concious ambulatory dogs, subjected to acute ischemia at a site remote from a previous infarction, clofilium was ineffective in preventing sudden death due to ventricular fibrillation. Figure 4 depicts the mortality curves and table 4 summarizes the electrophysiologic changes, infarct size, and thrombus mass data obtained from salinetreated control animals and animals treated chronically with clofilium. Of $10 \mathrm{dogs}$ given 2 $\mathrm{mg} / \mathrm{kg}$ clofilium followed by $1 \mathrm{mg} / \mathrm{kg}$ for $12 \mathrm{~h}, 7$ dogs succumbed to ventricular fibrillation within an average of $249 \pm 88 \mathrm{~min}$ after application of $150 \mu \mathrm{A}$ to the intimal surface of the left circumflux coronary artery. Each of the 10 salinetreated animals fibrillated $(173 \pm 45$ min-post 150 $\mu \mathrm{A})$ and the difference between these 2 groups of animals is not significant ( $P>0.10$, fig. 3 ).

Clofilium did produce a significant prolongation of the effective refractory period $(195 \pm 7 \mathrm{~ms}$ in clofilium-treated vs. $150 \pm 3 \mathrm{~ms}$ in saline controls, $\mathrm{P}<0.05$ ). However, this was not associated with a decrease in mortality in the clofilium-treated dogs suggesting that increasing refractoriness alone was not sufficient to prevent ventricular fibrillation in these animals.

Of the remaining parameters measured, clofilium-treated animals appeared slightly less susceptible to the production of sustained and non-sustained ventricular tachycardia, but this was not statistically significant. There were no significant differences between the thrombus mass, infarct size, or the time to electrocardiographic changes noted in table 4 in clofilium-treated and salinetreated dogs. These data suggest that clofilium exerted its principal electrophysiologic effect of prolongation of the effective refractory period, but failed to exert a significant protective antifibrillatory effect.

\section{TABLE 3}

Electrophysiological actions of clofilium in dogs subject to programmed electrical stimulation.

\begin{tabular}{|c|c|c|c|c|c|c|}
\hline \multirow[t]{2}{*}{ Dog } & \multicolumn{2}{|c|}{ Excitation threshold (mA) } & \multicolumn{2}{|c|}{$\begin{array}{l}\text { Effective refractory period } \\
\text { (ms; twice threshold } \mathrm{mA} \text { ) }\end{array}$} & \multicolumn{2}{|c|}{$\begin{array}{l}\text { Effective refractory period } \\
\text { (ms; } 10 \mathrm{~mA})\end{array}$} \\
\hline & Pre-clofilium & Post-clofilium & Pre-clofilium & Post-clofilium & Pre-clofilium & Post-clofilium \\
\hline 1 & 0.22 & 0.06 & 171 & 228 & 125 & 195 \\
\hline 2 & 0.05 & 0.07 & 176 & 175 & 125 & 115 \\
\hline 3 & 0.06 & 0.05 & 169 & 175 & 135 & 130 \\
\hline 4 & 0.06 & 0.08 & 181 & 185 & 150 & 145 \\
\hline 5 & 0.06 & 0.13 & 160 & 167 & 115 & 115 \\
\hline 6 & 0.11 & 0.11 & 198 & 190 & 155 & 120 \\
\hline \multicolumn{7}{|l|}{ Mean } \\
\hline \pm S.E.M. & $0.09 \pm 0.03$ & $0.08 \pm 0.01$ & $176 \pm 5$ & $187 \pm 9$ & $134 \pm 6$ & $137 \pm 13$ \\
\hline
\end{tabular}


TABLE 4

Action of clofilium in a canine model of sudden coronary death $^{\mathrm{a}}$.

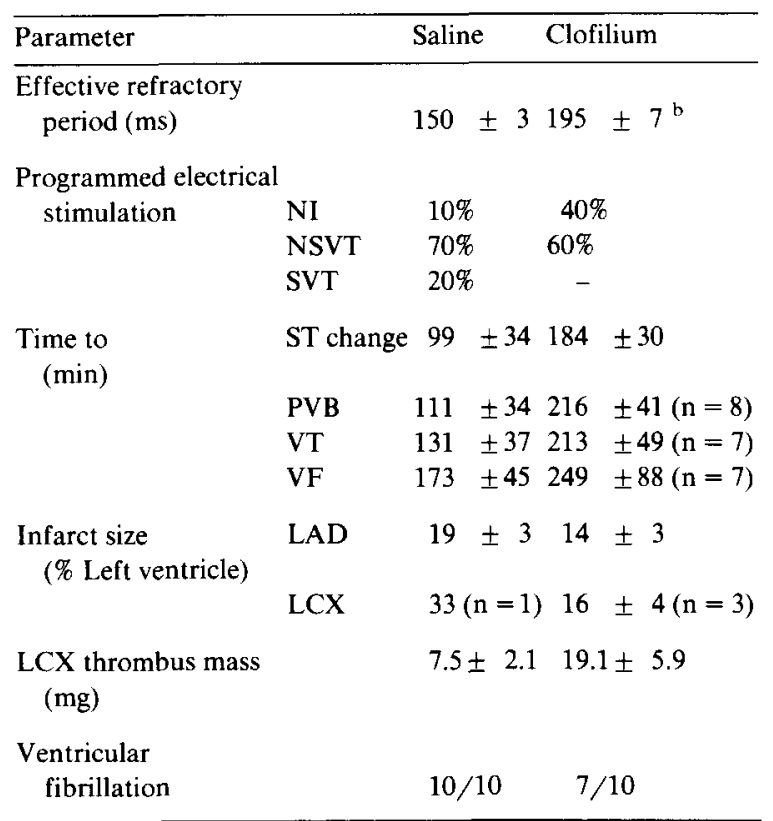

a NI, non-inducible; NSVT, non-sustained ventricular tachycardia; SVT, sustained ventricular tachycardia; PVB, premature ventricular beats; VT, ventricular tachycardia; VF, ventricular fibrillation; LAD, left anterior descending coronary artery; LCX, left circumflex coronary artery. ${ }^{\mathrm{b}} \mathbf{P}<0.05$ by non-paired $t$-test; $n=10$ except where otherwise noted.

One surprising and unexpected finding in this study was that clofilium appeared to occasionally produce ventricular arrhythmias. While these were not quantitated, 2 of the 7 anesthetized dogs subjected to programmed electrical stimulation and 6 of the 10 conscious dogs used in the sudden death model responded to clofilium administration with ventricular arrhythmias, characterized by both single and multiple multifocal premature complexes and occasionally short runs of spontaneous non-sustained ventricular tachycardia. These arrhythmias occurred in two dogs anesthetized acutely after administration of clofilium, and also were present in the conscious animals $12 \mathrm{~h}$ after clofilium administration.

\section{Discussion}

Mortality as a result of sudden coronary death represents a major cause of loss of life in the United States today. Estimates are that as many as 199000 to 386000 individuals (Doyle, 1979) may die suddenly each year. Because most of these deaths result from ventricular fibrillation secondary to ischemic heart disease (Peter et al., 1980), much experimental effort has been aimed at finding effective pharmacologic agents for the prevention of ventricular fibrillation. Clinical trials with the $\beta$-adrenergic blocking agents timolol, metoprolol and propranolol have demonstrated that these drugs are capable of reducing mortality due to sudden coronary death in individuals with a previous myoardial infarction. The precise mechanism by which this is accomplished, however, is not yet certain, although $\beta$-adrenergic blockade most likely plays an important role.

Previous investigators have found that the adrenergic neuronal blocking agent, bretylium, was effective in elevating the electrical ventricular fibrillation threshold in animals (Bacaner, 1968; Bacaner and Schrienemachers, 1968; Kniffen et al., 1975; Cervoni et al., 1971) and later work demonstrated bretylium to be efficacious as an antifibrillatory agent in man (Holder et al., 1977; Sanna and Arcidiacono, 1973). The mechanism of bretylium's antifibrillatory action, however, was believed not to be due to its adrenergic neuronal blocking properties since: (1) another adrenergic neuron blocking drug, guanethidine, was ineffective in elevating ventricular fibrillation threshold (Bacaner, 1968; Cervoni et al., 1971); (2) in vitro electrophysiologic studies demonstrated that bretylium exerted its primary electrophysiologic property (prolongation of action potential duration and tissue refractoriness) despite prior sympathetic blockade with either reserpine pretreatment (Wit et al., 1970) or immunosympathectomy (Namm et al., 1975); and (3) the in vivo electrophysiologic and antifibrillatory actions of bretylium were intact despite prior reserpine administration (Cervoni et al., 1971) or surgical denervation (Waxman and Wallace, 1972). The above observations suggest that prolongation of the ventricular action potential duration (and ventric- 
ular refractoriness) is bretylium's principal mechanism of action. Drugs which similarly prolong ventricular refractoriness may be effective in preventing ventricular fibrillation or reentrant arrhythmias (Steinberg and Molloy, 1979; Moe, 1975). Others have suggested that ventricular fibrillation is fundamentally a reentrant-type arrhythmia (Moe, 1975) where prolongation of the reentrant pathway by increasing refractoriness represents a reasonable approach to the prevention of ventricular fibrillation.

Recently, the new quaternary ammonium compound, clofilium, has been found to prolong action potential duration and myocardial refractoriness (Steinberg and Molloy, 1979; Steinberg et al., 1981). Clofilium produces a rate-dependent increase in action potential duration, increases the effective refractory period in proportion to the action potential duration, and appears to produce a greater increase in action potential duration in normal vs. acutely infarcted myocardium, thus decreasing the disparity in refractoriness of normal and infarcted tissue (Steinberg et al., 1981). Clofilium, however, has no effect on conduction, $V_{\max }$, and unlike bretylium, has no adrenergic neuronal blocking properties (Steinberg and Molloy, 1979). In addition to the above in vitro effects, clofilium has been shown to elevate electrical ventricular fibrillation threshold in dogs (Steinberg and Molloy, 1979) and prevents ventricular tachycardia and ventricular fibrillation in dogs subjected to programmed electrical stimulation (Michelson et al., 1981). These observations are in agreement with data presented in this report. Clofilium was fully effective, although at somewhat higher doses than those used elsewhere (Steinberg and Molloy, 1979; Platia and Reid, 1980; Greene et al., 1981; Michelson et al., 1981), in elevating electrical ventricular fibrillation threshold in both normal and previously infarcted canine myocardium and in preventing induced ventricular tachycardia and fibrillation in dogs undergoing programmed electrical stimulation. Additionally, clofilium was effective in slowing the rate of the tachycardia in inducible animals.

Clofilium was not, however, effective in a recently described model of sudden coronary death (Patterson et al., 1982). This model overcomes some of the objections raised with other animal models of ventricular arrhythmias and fibrillation. It is characterized by the presence of an area of previous myocardial infarction and the ability of programmed electrical stimulation to elicit reentrant ventricular arrhythmias. Drugs are evaluated in the conscious animal, thus eliminating the confounding effects of anesthesia upon both the autonomic nervous system and the myocardial electrophysiologic properties. In addition, ventricular dysrhythmias develop spontaneously as a result of either a partial or complete occlusion of the left circumflex coronary artery by a platelet thrombus (Patterson et al., 1982; Romson et al., 1980), resulting in mortality rates up to $100 \%$. When a similar thromboembolic lesion is produced in animals without previous infarction, the mortality is $20 \%$ (Patterson et al., 1982). Therefore, in the model employed in the present report, ventricular fibrillation, is produced by a generally non-lethal ischemic event. While clofilium was effective in increasing the duration of the effective refractory period over that of control animals, no significant decline in mortality was observed in this model of sudden death, although the trend for clofiliumtreated animals was favorable. In such studies with small numbers of animals there is always the risk that the failure of clofilium treatment was due to a type II statistical error viz., clofilium really is beneficial in such a model but the use of too few animals failed to show a statistical benefit. While this is certainly a consideration it is important to point out that other drugs such as bretylium (Holland et al., 1983) nadolol (Patterson and Lucchesi, 1983) amiodarone (Patterson et al., 1983) pranolium (Eller et al., 1983) and sotalol (Lynch et al., 1985) have all been studied in an identical model with the same numbers of animals in each treatment group and have shown significant benefits when compared to appropriate vehicle control animals. Thus, a relative comparison would suggest that clofilium is less effective in the canine model of sudden death than these other agents, yet there is clearly a favorable trend. These pharmacologic agents, however, have multiple actions. As previously mentioned, in addition to increasing myocardial cellular refractoriness, bretylium is also an adrenergic neuronal blocking agent. Con- 
versely, nadolol has little effect on conduction and refractoriness in myocardial tissue (Patterson and Lucchesi, 1982), but is a $\beta$-adrenergic blocking drug (Lee et al., 1975). Pranolium (UM-272) not only increases cellular refractoriness, but also decreases myocardial conduction both in vitro (Rosen et al., 1975) and in vivo (Gibson et al., 1978; Patterson and Lucchesi, 1981). Thus, the observation that clofilium failed to exert a significant antifibrillatory effect in the conscious dog suggests that, at least in this model, the milieu of factors which predisposes the heart to spontaneous ventricular fibrillation may require greater appeasement than simple prolongation of action potential duration and refractoriness. The underlying electrophysiological substrate during myocardial ischemia which ultimately provokes ventricular fibrillation is most likely the sum total of many individual factors working in concert. Even though clofilium is effective in prolonging the action potential duration in normal and ischemic myocardium in vitro, and is able to elevate electrical ventricular fibrillation threshold in vivo, during the dynamic process which ultimately leads to sudden death, one or a multiple of these factors may overcome any beneficial effect on action potential duration which has been produced by clofilium. So, while it is difficult to draw any firm conclusion based on the small number of animals used in the sudden death study, it is clear that further effort should be made to determine the precise benefit, if any, of prolongation of refractoriness in in vivo animal models of sudden coronary death.

In this regard, data from our study must question the benefit of prolonged refractoriness by clofilium as it regards clofilium's antifibrillatory effect. We saw no clear relationship between these two effects. In the VFT study, in the non-ischemic canine heart, clofilium clearly elevated myocardial ERP but at a dose which had no effect on VFT. Conversely, in chronically infarcted animals, clofilium produced no significant effect on ERP of normal myocardium but elevated the VFT to the same level as in the non-ischemic canine heart. This may be explained partially by the fact that the average paced heart rate in the non-ischemic group was somewhat lower than the paced rate in the infarcted group $(163 \pm 8$ vs. $190 \pm 6$ beats $/ \mathrm{min}$, respectively; $\mathrm{P}<0.05)$. However, in those animals subjected to programmed electrical stimulation, no change in ERP occurred after clofilium and the paced heart rate was $161 \pm 9$ beats/min, a value similar to the paced rate in the non-infarcted VFT group where a significant increase in ERP occurred after clofilium. As previously mentioned, while the ERP was prolonged in the animals studied under conditions which provoked sudden death, certainly this did not provide any protection to this group of animals. While others have reported a relationship between clofilium's antiarrhythmic and electrophysiologic action (Platia and Reid, 1980; Greene et al., 1981; Michelson et al., 1981), our data can not confirm this relationship.

It should be pointed out that in no animal can clofilium administration be causally related to either ventricular fibrillation or sudden death. However, any further evaluation of this agent should be tempered with the knowledge that ventricular arrhythmias may be an undesirable side effect of therapy with clofilium.

It should also be pointed out that limitation of the present study relates to the sampling sites chosen for electrophysiologic investigation. In general, sampling sites were in normal regions or regions bordering ischemic areas, but no direct measurements were made from ischemic areas. To the extent that ischemia generates electrophysiologic disturbances in non-ischemic tissues our observations are valid, especially since each dog was its own control. However, further studies in ischemic tissues are warranted especially since the apparent lack of correlation between clofilium's electrophysiologic and antiarrhythmic actions might be further clarified if ischemic tissue were studied.

In summary, clofilium is a new quaternary ammonium antiarrhythmic agent which is capable of elevating electrical ventricular fibrillation threshold in both normal and chronically infarcted dogs and prevents the induction of reentrant ventricular arrhythmias in dogs undergoing programmed electrical stimulation. It does not, however, significantly reduce mortality in a canine model of sudden coronary death at the dose studied. Although 
clofilium showed antiarrhythmic activity in the models studied, a significant protective effect was not observed in the sudden death model. This may indicate that a subtherapeutic drug level was achieved and a small increment in dose or change in schedule might show greater efficacy. Also, anesthetic effects may also account for this discrepancy. There is, however, a suggestion that a change in the ERP of normal myocardium induced by clofilium is not the sole mechanism of action of this drug.

\section{Acknowledgements}

The authors wich to express their grateful appreciation to Mr. Thomas Hess for his excellent technical assistance, and to Ms. Dixie Thomas, Ms. Pat Quiel and Ms. Kim Kanitz for their careful preparation of this manuscript.

\section{References}

Bacaner, M.B., 1968, Quantitative comparison of bretylium with other antifibrillatory drugs, Am. J. Cardiol. 21, 504.

Bacaner, M.B. and D. Schrienemachers. 1968, Bretylium tosylate for suppression of ventricular fibrillation after experimental myocardial infarction, Nature (London) 220, 494.

Beta-blocker Heart Attack Study Group, 1981, The $\beta$-blocker heart attack trial, JAMA 246, 2073.

Cervoni, P., C.H. Ellis and R.A. Maxwell, 1971, The antiarrhythmic action of bretylium in normal reserpine pretreated and chronically denervated dog hearts, Arch. Int. Pharmacodyn. Ther. 190, 91.

Dixon, W.J. and F.J. Massey, 1969. Introduction to statistical analysis (McGraw-Hill, New York), p. 167.

Doyle, J.T.. 1979. The risk of sudden death: the general population, NIH Working Group on Arteriosclerosis, Bethesda, MD.

Eller, B.T., E. Patterson and B.R. Lucchesi, 1983, Ventricular fibrillation in a conscious canine model - its prevention by UM-272, European J. Pharmacol. 87, 407

Gibson, J.K., J.L. Burmeister and B.R. Lucchesi, 1978, Electrophysiologic effects of UM-272 on myocardial ischemia in the canine heart, J. Pharmacol. Exp. Ther. 207, 304.

Greene, H.L., J.A. Werner, B.R. Gross, G.M. Kime, G.B. Trobaugh and L.A. Cobb, 1981. Selective prolongation of cardiac refractory times in many by clofilium, a new antiarrhythmic agent, Circulation (Abst.) 64, 137.

Hjalmarson, A., D. Elmfeldt, J. Herlitz et al., 1981, Effect on mortality of metoprolol in acute myocardial infarction, Lancet, II (8251), 823.

Holder. D.A., A.D. Sniderman. G. Fraser and E.L. Fallen. 1977. Experience with bretylium tosylate by a hospital cardiac arrest team, Circulation 55, 541.
Hollañd, K., E. Patterson and B.R. Lucchesi, 1983. Prevention of ventricular fibrillation by bretylium in a conscious canine model of sudden coronary death, Am. Heart J. 105. 711.

Kniffen, E.J., T.E. Lomas, R.E. Counsell and B.R. Lucchesi. 1975. The antiarrhythmic and antifibrillatory actions of bretylium and its o-iodobenzyltrimethylammonium analog, UM-360, J. Pharmacol. Exp. Ther. 192, 120.

Lee, R.J., D.B. Evans, S.H. Baky and R.J. Laffan, 1975 , Pharmacology of nadolol (SQ 11725), a new $\beta$-adrenergic antagonist lacking direct myocardial depression, European J. Pharmacol. 33, 371.

Lynch, J.J., C.A. Coskey, D.G. Montgomery and B.R. Lucchesi, 1985, Prevention of ventricular fibrillation by dextrorotatory sotalol in a conscious canine model of sudden death, Am. Heart J. 109, 949.

Michelson, E.L., M. Naito. D. David, L.S. Dreifus and E.N. Moore, 1981, Antiarrhythmic efficacy and electropharmacology of clofilium in a chronic canine ventricular tachyarrhythmia model, Circulation 64, 124.

Michelson, E.L., J.F. Spear and E.N. Moore, 1980, Electrophysiologic and anatomic correlates of sustained ventricular tachyarrhythmias in a model of chronic myocardial infarction, Am. J. Cardiol. 45, 583.

Moe, G.K., 1975, Evidence for reentry as a mechanism of cardiac arrhythmias. Rev. Physiol. Biochem. Pharmacol. 72, 55.

Namm, D.H., C.M. Wang. S. El-Sayad, F.C. Copp and R.A. Maxwell, 1975. Effects of bretylium on rat cardiac muscle: the electrophysiological effects and its uptake and binding in normal and immunosympathectomized rat hearts, $\mathbf{J}$. Pharmacol. Exp. Ther. 193, 194.

Norwegian Multicenter Study Group, 1981. Timolol-induced reduction in mortality and reinfarction in patients surviving acute myocardial infarction. New Eng. J. Med. 304, 801.

Patterson, E., B.T. Eller, G.D. Abrams. J. Vasiliades and B.R. Lucchesi, 1983, Ventricular fibrillation in a conscious canine preparation of sudden coronary death - prevention by shortand long-term amiodarone administration, Circulation 68 . 857.

Patterson, E., K. Holland, B.T. Eller and B.R. Lucchesi, 1982, Ventricular fibrillation resulting from ischemia at a site remote from previous myocardial infarction - a conscious canine model of sudden coronary death, Am. J. Cardiol. 50 , 1414.

Patterson, E. and B.R. Lucchesi, 1981, Chronic ventricular tachyarrhythmias in the conscious dog: prevention by UM-272 (dimethylpropranolol), J. Cardiovasc. Pharmacol. 3. 769 .

Patterson. E. and B.R. Lucchesi, 1982, Antifibrillatory actions of nadolol, J. Pharmacol. Exp. Ther. 223, 144.

Patterson, E. and B.R. Lucchesi, 1983, Antifibrillatory actions of $\mathrm{d}, \mathrm{l}$-nadolol in a conscious canine model of sudden coronary death, J. Cardiovasc. Pharm. 5, 737.

Peter, T., H. Hamamoto, J. Jordon, M. Platt and W. Mandel, 1980. Indications for antiarrhythmic therapy as prophylaxis against sudden death, in: Cardiac Arrhythmias: Mechanisms and Management, ed. A. Castellanos, Cardiovascular Clinics, p. 249 
Platia, E. and P.R. Reid, 1980, Suppression of induced ventricular tachycardia by the quaternary ammonium, clofilium, Circulation (Abst.) 62, 153.

Romson, J.L., D.W. Haack and B.R. Lucchesi, 1980, Electrical induction of coronary artery thrombosis in the ambulatory canine: A model for in vivo evaluation of antithrombotic agents, Thromb. Res. 17, 841.

Rosen, M.R., D.S. Miura and P. Danilo, 1975, The effects of dimethyl quaternary propranolol on the electrophysiologic properties of canine cardiac Purkinje fibers, J. Pharmacol. Exp. Ther. 193, 209.

Sanna, G. and R. Arcidiacono, 1973, Chemical ventricular defibrillation of the human heart with bretylium tosylate, Am. J. Cardiol. 32, 982.
Steinberg, M.I. and B.B. Molloy, 1979, Clofilium: A new antifibrillatory agent that selectively increases cellular refractoriness, Life Sci. 25, 1397.

Steinberg, M.I., M.E. Sullivan, S.A. Wiest, F.W. Rockhold and B.B. Molloy, 1981, Cellular electrophysiology of clofilium, a new antifibrillatory agent, in normal and ischemic canine Purkinje fibers, J. Cardiovasc. Pharmacol. 3, 881.

Waxman, M.B. and A.G. Wallace, 1972, Electrophysiological effects of bretylium tosylate on the heart, J. Pharmacol. Exp. Ther. 183, 264.

Wit, A.L., C. Steiner and A.N. Damato, 1970, Electrophysiologic effects of bretylium tosylate on single fibers of the canine specialized conducting system and ventricle, J. Pharmacol. Exp. Ther. 173, 344. 